Revista Búsqueda, Vol. 4 Núm. 19:158-175; 2017. ISSN: 0123-9813

\title{
El desentrenamiento: un subsistema necesario en la preparación del deportista cubano
}

\author{
Detraining: a necessary subsystem in the Cuban sportsman \\ preparation
}

\author{
Rolando Zamora-Castroํㅜ Noralmis Frómeta-Moreira²
}

\begin{abstract}
${ }^{1}$ Doctor en Ciencias Pedagógicas, Licenciado en Cultura Física. Docente-investigador de la Facultad de Cultura Física, Universidad de Oriente, Santiago de Cuba, Cuba. Identificador de autor: https://orcid. org/0000-0001-7293-6770; correo: rzamora@uo.edu.cu

${ }^{2}$ Doctora en Ciencias de la Cultura Física, Licenciada en Cultura Física. Docente-investigadora de la Facultad de Cultura Física, Universidad de Oriente, Santiago de Cuba, Cuba. Identificador de autor: https://orcid. org/0000-0003-1354-9352; correo: nfrometam@uo.edu.cu
\end{abstract}

Recibido: 05-10-2017; Revisado: 28-10-2017; Aceptado: 13-11-2017

\section{Resumen}

El objetivo de este artículo consistió en elaborar una estructura sistémica, donde se integre por primera vez el desentrenamiento a la «preparación del deportista» cubano, pues hasta ahora está separado de la misma. La original propuesta de «periodización del entrenamiento deportivo» de Matveev (1977), se tomó como el principal referente de este estudio, aunque después de ese hecho han surgido otros modelos de entrenamiento más a tono con las exigencias competitivas actuales en el mundo. Autores como Morales (2016), Pino (2014), Mena, (2014), Mustelier (2014), Pérez y Soria (2014), Mayo (2012), Crusellas (2012), Pérez (2012) y otros, estudiar al desentrenamiento desde las perspectivas médicas o metodológicas, pero ninguno lo reconoció como parte de la «preparación del deportista». Ese vacío filosófico se aprovechó en este estudio y así contribuir a propiciar su conocimiento teórico y práctico. Entre los métodos teóricos se emplearon el análisis-síntesis y el hipotético-deductivo, de los empíricos, el sistémico-estructural-funcional, el taller de familiarización. Entre los resultados más sobresalientes se destacaron la conciliación de las opiniones de los comisionados del deporte de Santiago de Cuba, quienes por primera vez reconocieron sus obligaciones éticas y humanistas en ser partícipes en la preservación de la salud de sus deportistas al cesar sus rutinas de modo definitivo. La conclusión más representativa de este artículo consistió en agregar una estructura capaz de equilibrar los efectos del entrenamiento dentro de la propia «preparación del deportista», elevando así su carácter ético-humanista.

Palabras clave: preparación del deportista, periodización del entrenamiento, desentrenamiento deportivo. 


\section{Abstract}

The objective of this article was to elaborate a systemic structure, where for the first time the detraining was integrated into the Cuban «athlete's preparation», since until now it has been separated from it. Matveev's original «periodization of sports training» (1977) was taken as the main reference in this study, although after that fact other training models have arisen more in tune with the current competitive demands in the world. Authors such as Morales (2016), Pino (2014), Mena, (2014), Mustelier (2014), Perez and Soria (2014), May (2012), Crusellas (2012), Perez (2012) and others, study the detraining from the medical or methodological perspectives, but none of them recognized it as part of the "preparation of the athlete". This philosophical vacuum was used in this study and thus contribute to propitiate their theoretical and practical knowledge. Among the theoretical methods used were the analysis-synthesis and the hypothetical-deductive, the empirical, the systemic-structural-functional, the familiarization workshop. Among the most outstanding results were the conciliation of the opinions of the sports commissioners of Santiago de Cuba, who for the first time recognized their ethical and humanist obligations to be participants in the preservation of the health of their athletes by stopping their routines so definitive. The most representative conclusion of this article was to add a structure capable of balancing the effects of training within the "preparation of the athlete", thus elevating its ethicalhumanistic character.

Key words: athlete's preparation, sport training periodization, sport detraining.

\section{Introducción}

Para propiciar la comprensión del problema tratado en este trabajo, es conveniente primero aclarar que sus autores no consideran sinónimos los conceptos «preparación del deportista» y «entrenamiento deportivo». Esa misma posición la asumió Matveev (1977), cuando escribió su teoría sobre la «periodización del entrenamiento deportivo», no obstante, aún en Cuba no pocos entendidos de manera errónea tienden a asemejarlos, a pesar de ser "...el primero (...) mucho más amplio" (p.7) y eso ha dificultado acertar en la organización sistémica de esos conceptos y otros muy asociados a los mismos.

Matveev (1977), incluyó en la «preparación del deportista» "...todos los medios que pueden contribuir a la consecución y mejora de la capacidad de rendimiento deportivo" (p.7), así como los educativos. Sin embargo, aquí no se comparte su idea, pues al desconocer al proceso de desentrenamiento como parte de esa preparación se simplificó su complejidad, dándole en cambio, gran importancia al entrenamiento deportivo, definiéndolo como un "...proceso ininterrumpido de varios años que se distingue por su carácter cíclico (un ciclo, más o menos perfecto, de determinados períodos). Por eso, llamó «periodización del entrenamiento» al cambio periódico y regular de la estructura y del contenido del entrenamiento dentro de un ciclo determinado" (pp.7-8). Él abordó unos de los "...problemas de la teoría y la praxis del deporte..." (p.7), y no le faltó razón, pues en Cuba después de haberse utilizado durante muchos años su propuesta, en la actualidad esta perdura en el deporte escolar de base, no así en el alto rendimiento, hoy permeado por otros modelos para dar respuesta a las nuevas exigencias competitivas internacionales. 
Fue el primero en concebir esa idea en el mundo, de ahí su importancia teórica y metodológica en este trabajo, además, de percibirse en su periodización el origen del desentrenamiento deportivo.

En la Teoría y Metodología del Entrenamiento Deportivo, en general, se sistematizan muchos conceptos derivados de las aportaciones de importantes estudiosos como Verkhoshansky (2002), Harre (1989), Platonov, (1987), Matveev (1977), Ozolin (1970), Bompa (s.a) y otros, donde existe bastante coincidencia en definir al entrenamiento como "... un proceso sistemático repetitivo $y$ progresivo de ejercicios, teniendo como objetivo el mejoramiento de la performance atlética" (Bompa, s.a., p.7), e implica "...la selección y uso efectivo de los medios, métodos y procedimientos (...) la obtención de la forma deportiva..." (Barrios y Ranzola, 1998, p.13). Este usa el ejercicio físico regular para causar adaptaciones fisiológicas y la medra del rendimiento deportivo, pero su reducción ocasiona la pérdida parcial o completa afectando a este último, al ocurrir "... alteraciones en la actividad hormonal y cambios en la función muscular y neural" (Andrade, 2009, p.1).

En particular, se ha avanzado mucho en los saberes del entrenamiento y a ellos se deben en gran medida, por solo citar un ejemplo, los fantásticos resultados exhibidos en la primera década del siglo XXI por el ex-velocistas jamaicanos Usain Bolt, quien legó a la humanidad en las carreras de $100 \mathrm{~m}$ y $200 \mathrm{~m}$ planos los astronómicos registros mundiales de $9,58 \mathrm{~s}$ y $19,19 \mathrm{~s}$ respectivamente. No obstante, ahora la cuestión está en cómo garantizarle un adecuado programa de desentrenamiento, para contrarrestar las adaptaciones morfo-funcionales y psicológicas que los mantuvieron en el estrellato olímpico y mundial casi una década. Pero el problema está en que los conocimientos existentes en esa dirección, no han tenido tanta suerte como los de su opuesto. Además, no se posee claridad en ese sentido, porque todavía no hay consenso en incluir al proceso de desentrenamiento dentro de la «preparación del deportista». Eso representa para los autores de este artículo un serio desacierto teóricopráctico en la actualidad, por ello, este estudio se enfocó solo en los rasgos generales de la «periodización del entrenamiento deportivo», definida por Matveev (1977), y esto no es casual. Cuando él propuso el «período de transición» o de tránsito -como se le llamó en Cuba- e incluso los descansos dentro de las sesiones de entrenamiento, reconocía la existencia de limitaciones biológicas en los deportistas para el trabajo físico.

El desentrenamiento deportivo no ha estado exento de definiciones no siempre acertadas, por un lado, y por otro, no son aceptadas por completo, a pesar de compartir con su contraparte la misma base léxica. Alonso (2000), calificó al desentrenamiento "...como un proceso médico-pedagógico (...) en correspondencia con el inverso del proceso de entrenamiento, al apuntar o enfatizar en evitar o disminuir los problemas de salud asociados al entrenamiento y lograr la readaptación a la vida cotidiana" (p.2) del ex-deportista, cambiada durante varios años, por otra de férrea disciplina, grandes privaciones y un riguroso régimen de trabajo físicodeportivo, el cual ocasionará ciertas adaptaciones morfo-funcionales y psicológicas según la exigencia de cada modalidad practicada.

El Instituto de Medicina del Deporte (IMD), inconforme con la situación del desentrenamiento en las preselecciones nacionales, elaboró un "Programa Nacional para la Atención a Ex- 
deportistas" (IMD, 2000). Este no pasó de ser una excelente idea, porque no se generalizó en el país. En este se excluyeron a los salidos de las Academias, Escuelas de Perfeccionamiento Atlético (ESPA) e Integral Deportiva (EIDE) de las provincias. Aun muchos de ellos, se diluyen en el tejido social de sus comunidades, fuera del control médicopedagógico correspondiente para prevenirlos de algún trastorno de salud, derivado del entrenamiento de alto rendimiento, Eso lo ratificó Pino (2014), Director del IMD, cuando informó en el "Balance Nacional de Atención a Atletas" lo siguiente: "...el $40 \%$ de los retirados hasta el 2013 no llegaron a los 60 años, cuando como paradoja el promedio de vida de hombres y mujeres en Cuba es 75 y 78 años en ese orden". Para esa fecha, a manera de ejemplo, ya habían fallecido varios ex-boxeadores por problemas cardíacos, como Jorge Guzmán -50 años (†2011)-, Teófilo Stevenson -60 años (†2012)- y Arnaldo Mesa -43 años (†2013). Por eso, el desentrenamiento deportivo ya es en un problema social.

El Instituto Nacional de Deportes, Educación Física y Recreación (Inder), como órgano rector de esas actividades, en el curso 2009-2010 se pronunció por "...continuar fortaleciendo la labor médica y psicológica deportiva con los atletas y el desarrollo de la educación antidoping" (Inder, 2009, p.15), sin embargo, no se refirió al problema del desentrenamiento. Eso demostró la existencia de una pobre visualización de ese proceso, en un país donde el deporte al pasar a ser un derecho de la población desde 1959, se convirtió en la principal cantera de sus campeones olímpicos, mundiales, panamericanos y centroamericanos y del Caribe.

En Cuba las investigaciones sobre el desentrenamiento se han venido incrementando de manera gradual, a pesar de ello, hasta el momento no se ha reparado en la necesidad de definir su naturaleza y límites, no obstante, autores como Morales (2016), Pino (2014), Mena (2014), Mustelier (2014), Pérez y Soria (2014), Mayo (2012), Crusellas (2012), Pérez (2012), Barroso (2010), Mujica (2010), Navarro (2010), Andrade (2009), Carvajal, (2009) y otros, lo han estudiado desde diferentes visiones. Algunos diagnosticaron a deportistas retirados de repente y no desentrenados, hallando dificultades de salud parecidas entre los de una misma disciplina; Aguilar (2009), Díaz (2005) y Barrizonte y otros (2003), descubrieron enfermedades como isquemia cardiaca, hipertensión arterial, anemia, hepatitis, obesidad y taquicardia. Carvajal (2009), se apoyó en esos antecedentes para proponer un programa de desentrenamiento, cuando también halló algunos de esas situaciones en ex-beisbolistas en Santiago de Cuba.

¿Por qué un médico debe encargarse de la atención al ex-deportista?, ¿retirarse del deporte es sinónimo a estar enfermo?, ¿si todo deportista de alto rendimiento, merece ser desentrenado, quién asume esa tarea y dónde se forma para tal fin? De modo invariable, todas las respuestas apuntan a no existir una idea clara sobre el lugar ocupado por el proceso de desentrenamiento y es como si su existencia, al parecer, estuviera generada por otro hecho distinto al entrenamiento. Ignorar la concatenación universal de los fenómenos de la naturaleza, la sociedad y el pensamiento, a veces, dificulta su total comprensión. Por tanto, el rol del médico del deporte aquí no se sobredimensiona, porque se le reconoce en el desentrenamiento el mismo lugar ocupado en el entrenamiento deportivo: prevenir las alteraciones de la salud, sin olvidar que sus métodos no son propios de la «preparación del deportista».

El razonamiento de los autores para elaborar este artículo fue simple, 
pues partieron de considerar según la Teoría General de Sistemas creada por Bertalanffy (1986), donde se asegura que en cualquiera de esas entidades todas las funciones de sus componentes contribuyen a la consecución del objetivo del ente de orden mayor, por tanto, no se pueden confundir en el deporte los conceptos "preparación" y "entrenamiento", y si se trata de graficar esa relación, en el mejor de los casos el entrenamiento se subordinará al primero, siendo eso una primera relación.

Ningún sistema está compuesto por un solo subsistema. En Cuba es común oír sistema de «preparación del deportista», al cual se le reconoce como único componente el entrenamiento. Este error no resiste el análisis sistémico. La otra cuestión es que en este artículo se desecha la idea de Matveev (1977) de considerar al «período de transición» dentro del subsistema de entrenamiento, porque sus medios y métodos se orientan a la recuperación del deportista a partir de la disminución del volumen y la intensidad del trabajo. Si todo esto es así, entonces, ¿cómo ese período contribuye al logro del objetivo del sistema mayor, si su función se diferencia de los fines de los períodos preparatorio y competitivo? Esto muestra la existencia de un serio problema de conocimiento a resolver mediante el diagnóstico de los directivos del deporte, al menos en Santiago de Cuba, partiendo de la presunción que ambos procesos se dan en una relación dialéctica contradictoria y de mutua exclusión, en tanto, la existencia de uno la determinada el otro. Además de considerar su ubicación dentro de un mismo sistema: la «preparación del deportista».

En resumen, a partir de asumir las prerrogativas del pensamiento divergente o lateral, para dar una solución alternativa diferente al problema de no considerarse aún al desentrenamiento dentro de la «preparación del deportista» en Cuba, originado tal vez, por la arraigada influencia de la «periodización del entrenamiento deportivo» de Matveev (1977), el objetivo de este artículo consistió en elaborar una estructura sistémica, donde se integre por primera vez el desentrenamiento a la «preparación del deportista» cubano, por su carácter social, ético y humanista, basado en el cumplimiento de "...los mismos principios del entrenamiento deportivo, con algunas interpretaciones y direcciones diferentes" (Alonso, Forteza, y López, 2001, p.3), porque las consecuencias funcionales y psicológicas derivadas del entrenamiento de alto rendimiento y la demanda de la sociedad cubana así lo exigen.

\section{Metodología}

Este estudio involucró una muestra (población) seleccionada de modo intencional no probabilística, compuesta por $33(100 \%)$ metodólogos o funcionarios, responsables de los deportes auspiciados por la Dirección Provincial de Deportes del Inder en Santiago de Cuba. Todos egresaron de la Carrera Licenciatura en Cultura Física. Ellos ofrecieron sus opiniones sobre diversas cuestiones relacionadas con el desentrenamiento, luego de participar en un taller de familiarización sobre ese tema. La experiencia colectiva en el cargo superó los siete años. Ellos se distribuyen en cinco agrupaciones como se muestra en la tabla 1 . 
Zamora-Castro et al. El desentrenamiento: un subsistema necesario en la preparación del deportista cubano

Tabla 1. Distribución de metodólogos por deportes. Provincia Santiago de Cuba

\begin{tabular}{|c|c|c|c|c|c|c|}
\hline No & Agrupaciones deportivas & & Deportes & Cantid & ólogos & $\%$ \\
\hline 1 & \multirow{6}{*}{$\begin{array}{l}\text { Juegos } \\
\text { Deportivos (1) }\end{array}$} & 1 & Balonmano & 1 & \multirow{6}{*}{6} & \multirow{6}{*}{18} \\
\hline 2 & & 2 & Béisbol & 1 & & \\
\hline 3 & & 3 & Fútbol & 1 & & \\
\hline 4 & & 4 & Hockey & 1 & & \\
\hline 5 & & 5 & Softbol & 1 & & \\
\hline 6 & & 6 & Voleibol & 1 & & \\
\hline 7 & \multirow{6}{*}{$\begin{array}{l}\text { Juegos } \\
\text { Deportivos (2) }\end{array}$} & 1 & Bádminton & 1 & \multirow{6}{*}{6} & \multirow{6}{*}{18} \\
\hline 8 & & 2 & Baloncesto & 1 & & \\
\hline 9 & & 3 & Polo acuático & 1 & & \\
\hline 10 & & 4 & Pelota Vasca & 1 & & \\
\hline 11 & & 5 & Tenis de Mesa & 1 & & \\
\hline 12 & & 6 & Tenis de Campo & 1 & & \\
\hline 13 & \multirow{10}{*}{$\begin{array}{l}\text { Tiempo y } \\
\text { Marcas }\end{array}$} & 1 & Atletismo & 1 & \multirow{10}{*}{10} & \multirow{10}{*}{30} \\
\hline 14 & & 2 & Ciclismo & 1 & & \\
\hline 15 & & 3 & Kayaks & 1 & & \\
\hline 16 & & 4 & Natación & 1 & & \\
\hline 17 & & 5 & Pentatlón-Triatlón & 1 & & \\
\hline 18 & & 6 & Pesas & 1 & & \\
\hline 19 & & 7 & Remos & 1 & & \\
\hline 20 & & 8 & Tiro Deportivo & 1 & & \\
\hline 21 & & 9 & Tiro con Arco & 1 & & \\
\hline 22 & & 10 & Velas & 1 & & \\
\hline 23 & \multirow{5}{*}{$\begin{array}{l}\text { Arte } \\
\text { Competitivo }\end{array}$} & 1 & Ajedrez & 1 & \multirow{5}{*}{5} & \multirow{5}{*}{15} \\
\hline 24 & & 2 & Clavados & 1 & & \\
\hline 25 & & 3 & Gimnasia Artística & 1 & & \\
\hline 26 & & 4 & Gimnasia Rítmica Deportiva & 1 & & \\
\hline 27 & & 5 & Nado Sincronizado & 1 & & \\
\hline 28 & \multirow{6}{*}{$\begin{array}{l}\text { Deportes de } \\
\text { Combate }\end{array}$} & 1 & Boxeo & 1 & \multirow{6}{*}{6} & \multirow{6}{*}{18} \\
\hline 29 & & 2 & Esgrima & 1 & & \\
\hline 30 & & 3 & Judo & 1 & & \\
\hline 31 & & 4 & Karate-Do & 1 & & \\
\hline 32 & & 5 & Lucha & 1 & & \\
\hline 33 & & 6 & Taekwondo & 1 & & \\
\hline & Totales & & 33 & 33 & 33 & 100 \\
\hline
\end{tabular}


Enesteestudioseefectuóunaaproximación al tema del desentrenamiento para propiciar la re-estructuración de la visión actual de la «preparación del deportista», dirigida a contribuir al completamiento de su perspectiva sistémica en Cuba. En ese sentido, se emplearon los siguientes métodos:

1. Análisis-síntesis:

permitió identificar las particularidades del desentrenamiento, para connotarlo en una nueva estructura a tono con su naturaleza pedagógica, así como evaluar las opiniones ofrecidas por los metodólogos investigados que participaron en el taller de socialización.

2. Revisión documental: se destinó al acopio de la mayor cantidad de información, para argumentar la necesidad de incorporar el llamado «periodo de transición» de Matveev (1977), a otra estructura, más a tono con sus funciones dentro de la propia «preparación del deportista».

3. Inductivo-deductivo: propició mediante un proceso de abstracción de lo general a lo particular y viceversa, concebir las posiciones y relaciones de subordinación de los componentes de la estructura a elaborar.

4. Sistémico-estructural funcional: se utilizó en la configuración de la estructura capaz de contener al desentrenamiento como otro subsistema de la «preparación del deportista», así como resistir su análisis desde una perspectiva sistémica.

5. Técnica de trabajo en grupo: se aplicó un taller de familiarización con los metodólogos estudiados. Al finalizar y para evaluar esa actividad se ejecutó el procedimiento de criterios PNIR (P-Positivos, N-Negativos, I-Interesantes, R-Recomendaciones); este consistió en analizar, sintetizar y procesar sus opiniones emitidas por escrito y de forma anónima sobre el desentrenamiento, siguiendo el significado de cada grafema inicial de la sigla PNIR. Esto propició conocer las tendencias de sus criterios y decidir, en general, si era válida o no, la idea de incluir al desentrenamiento dentro del proceso de «preparación del deportista» cubano

\section{Indicaciones para valorar los} criterios PNIR, sobre la necesidad de considerar al proceso de desentrenamiento deportivo dentro de la «preparación del deportista» cubano

Aquí se tuvo en cuenta lo siguiente. EI criterio «P» sobre un asunto particular tratado se significa como cierto, efectivo, verdadero y no da lugar a ninguna duda. Se aplica a lo consistente en la existencia de un hecho, fenómeno o, suceso real, útil o práctico y no a su falta. El «N» implica un franco rechazo, contradicción o disposición a ver y juzgar una situación concreta por el lado más desfavorable. El «I» se emite cuando se piensa, opina o considera un hecho atractivo, por su importancia para un fin, o resulta novedoso por su rareza o despierta la admiración por algo no visto ni oído antes en un contexto específico. La «R» es una idea portadora de la intención de demandar la toma de cierta disposición anticipada, conducente a logro de un objetivo particular.

Para no forzar la recolección de los datos PNIR, al concluir el taller de familiarización, se le solicitó a la muestra $(100 \%)$, plasmar en una hoja de papel sus criterios al respecto, sin tener la obligación de adicionarle ninguna forma conducente a su identificación personal. A tal petición accedieron solo 21 (64\%) metodólogos. Las respuestas abarcarían todos los significados contenidos en la sigla PNIR. Quienes optaron por no realizar este ejercicio de la técnica de grupo, se clasificaron como neutrales. 


\section{Resultados y discusión}

En la tabla 2 se muestra un resumen de los resultados del análisis de los criterios PNIR. Los criterios positivos (21) ofrecidos por los metodólogos sobre los aspectos sugeridos superaron a los negativos (12). En ningún caso se consideró absurda, sin sentido o improcedente, la idea de integrar al proceso de desentrenamiento dentro de la «preparación del deportista» cubano y así, poder reforzar su carácter ético y humanista. Sin embargo, las opiniones giraron en torno a las barreras cognitivas y filosóficas vigentes, erigidas como focos de resistencia negativa, al ignorar su capacidad para perfeccionar al deporte cubano en la actualidad. En los \% de respuestas, aunque al taller participaron los 33 (100\%) metodólogos estudiados (columnas 1 y 4 ), solo vertieron sus criterios positivos 21 (columna 1), cifra tomada como base (100\%) para determinar el \% del resto de las respuestas de la columna 2 . En las columnas 3 y 5 los porcientos se calcularon en base a 33 metodólogos.

Tabla 2. Resultados de la evaluación de los criterios PNIR de los metodólogos sobre la inclusión del desentrenamiento en la «preparación del deportista»

\begin{tabular}{|c|c|c|c|c|c|c|}
\hline \multicolumn{2}{|r|}{ Criterios } & \multirow{2}{*}{$\begin{array}{c}\begin{array}{c}\text { Cantidad de } \\
\text { criterios } \\
\text { (1) }\end{array} \\
21\end{array}$} & \multirow{2}{*}{$\begin{array}{c}\begin{array}{c}\text { \% de } \\
\text { respuestas } \\
\text { (2) }\end{array} \\
100\end{array}$} & \multirow{2}{*}{$\begin{array}{c}\begin{array}{c}\text { \% del } \\
\text { total } \\
(3)\end{array} \\
64\end{array}$} & \multirow{2}{*}{$\begin{array}{c}\text { No respondieron } \\
\mathbf{( 4 )}\end{array}$} & \multirow{2}{*}{ 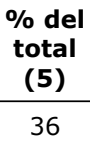 } \\
\hline 1 & Positivos (P) & & & & & \\
\hline 2 & Negativos (N) & 12 & 57 & 36 & 21 & 64 \\
\hline 3 & Interesantes (I) & 13 & 62 & 39 & 20 & 61 \\
\hline 4 & Recomendaciones ( $R$ ) & 11 & 52 & 33 & 22 & 67 \\
\hline
\end{tabular}

Nota: los porcientos (\%) son aproximados (por defecto o por exceso), pues se tomó en cuenta la característica discreta de los datos manejados

Si se les presta atención a los requerimientos del procedimiento PNIR (Ferreiro, 2003), pudiera pensarse en la existencia de un consenso en las opiniones opuestas a la inclusión del desentrenamiento dentro de la «preparación del deportista», porque ese contenido no estuvo incluido en sus programas de estudio en la Facultad de Cultura Física durante muchos años (desde el 2007 se le dedica el irrisorio tiempo de dos horas a ese tema en la asignatura Control Médico). Tal vez esa situación la provocó el desconocimiento al respecto. Aun así, 21 (64\%) metodólogos admitieron su necesidad $y$ responsabilizaron al Inder $y$ sus dependencias territoriales, incluyendo al
IMD con su red de centros provinciales con la dirección y ejecución de ese proceso, con la misma prestancia dispuesta para el entrenamiento, pues con el retiro del deportista de alto rendimiento, debe aumentar la observancia de su estado de salud.

A continuación, se exponen algunos resultados conseguidos con el PNIR, de donde se estratificaron las ideas válidas para ratificar, la necesidad de elaborar una estructura dirigida a asegurar los rasgos teóricos básicos sobre el desentrenamiento al ser incorporado a la «preparación del deportista», y le asegure igual nivel de importancia como se hace con el entrenamiento deportivo. 


\begin{abstract}
Necesidad de incorporar al desentrenamiento en la «preparación del deportista» cubano: análisis e interpretación de las respuestas dadas por la muestra estudiada
\end{abstract}

\section{Resultado 1: Positivos}

1. Integrar ese proceso al Sistema Deportivo Cubano como una exigencia, desde el deporte de alto rendimiento practicado en las provincias hasta las preselecciones nacionales, asumiendo la formación del personal capaz de conducirlo tanto en la base como en la nación.

2. Los entrenadores deben concientizar a sus deportistas sobre la importancia de incorporarse a un programa de desentrenamiento al retirarse, desde el comienzo de sus vidas en el deporte, sin considerar la etapa por donde transiten.

3. Por su relevancia y especificidad de sus tareas, es vital diseñar su propia teoría y metodología, al ser sus objetivos y contenidos diferentes a los del entrenamiento deportivo.

4. Su novedad requiere una mayor profundización desde la ciencia, para evitar por su desconocimiento la ocurrencia de fallecimientos entre los ex-deportistas, asociadas a las adaptaciones morfo-funcionales sufridas en sus carreras.

5. Incorporar al desentrenamiento como subsistema y de manera definitiva, dentro de la actual concepción de la «preparación del deportista» cubano, pues es la manera ideal para garantizar su retorno seguro a la vida cotidiana justo al poner fin a su paso por esa actividad.

6. Las comisiones provinciales y nacionales deben asumirlo como otra línea de su trabajo y darle mayor importancia, y así evitarle al exdeportista alguno de los problemas de salud causados por el entrenamiento de elite.
7. El desentrenamiento organizado y controlado, es la fórmula idónea para asegurarle la salud a quienes se retiren del deporte en Cuba.

8. La provincia debe preocuparse y ocuparse del desentrenamiento de sus deportistas, cuando estos dejen de ser entrenados para siempre y se dediquen a otras tareas sociales.

9. El desentrenamiento es una realidad inaplazable y un servicio vital en el deporte de hoy.

10. La Dirección Provincial de Deportes debe auspiciar y estimular las investigaciones científicas sobre el desentrenamiento en la Facultad de Cultura Física y el Centro Provincial de Medicina del Deporte.

Análisis e interpretación (criterios positivos)

Los criterios de los metodólogos examinados fueron satisfactorios, pues coincidieron (64\%) en la necesidad de integrar el desentrenamiento a la «preparación del deportista». Eso completaría su expresión ética y humanista, ya perpetuada al convertirse su práctica en un derecho del pueblo. Ese ideal, además, se consolida mediante la capacitación del personal para dirigirlo en los territorios. Al parecer, la complejidad y especificidad del desentrenamiento, demanda la creación de su propia teoría y metodología, con sus principios, métodos, procedimientos, formas de control y evaluación. Entre ellos existió coincidencia en la urgencia de garantizar de inmediato un retorno seguro a la vida cotidiana cuando el deportista se retira, al ser esto una demanda social y asumir con ello, al desentrenamiento como una nueva perspectiva, para el perfeccionamiento de la visión existente sobre el deporte, y admitirlo como el completamiento de la «preparación del deportista».

Fue gratificante observar como muchos metodólogos reconocieron en el 
desentrenamiento, la oportunidad de verse involucrados en un problema trascendental y actual de su profesión, no resuelto hasta el momento en Cuba. Por tanto, fue magnífico su apoyo para tratar de erigir en Santiago de Cuba un centro especializado para desarrollar ese proceso, como una muestra concreta del reconocimiento social a quienes se retiran del deporte activo.

\section{Resultado 2: Criterios negativos}

1. Se desconoce la proyección nacional sobre el impacto negativo en lo político, económico, social y económico, por no exigir el desentrenamiento de los deportistas al retirarse en el país.

2. Al no tenerse suficiente bibliografía y una teoría, su aplicación científica se dificulta.

3. En el pregrado y el posgrado no lo han asumido como algo vital. El entrenamiento de alto rendimiento con sus métodos causa modificaciones morfo-funcionales y psicológicas en el deportista para el gran resultado, pero no se enseña con la misma disposición las vías para preservar la salud, cuando aquel deja de ser un practicante sistemático, tampoco está claro quien responde, caso que alguna causa derivada del entrenamiento le provoque la muerte.

4. La falta de una concepción coherente, impide tener una comprensión más clara de la complejidad del esquema general de la «preparación del deportista» cubano.

5. Existen años de vacío en el conocimiento y su práctica, y eso es polémico, al no ser formados los egresados de Cultura Física, conociendo a profundidad la importancia del desentrenamiento.

6. El desentrenamiento en el país y Santiago de Cuba en particular, se caracteriza por la falta de conocimiento y de voluntad, para asegurar la infraestructura, los recursos humanos y financieros, para empezar a atender ese asunto de impacto social.

7. Por lo general, los entrenadores ignoran los riesgos producidos por el entrenamiento de alto rendimiento, ni cómo estos se pueden contrarrestar cuando el deportista se retira.

8. En Santiago deCubala responsabilidad del desentrenamiento recayó sobre el Centro de Medicina del Deporte, sin embargo, este no maneja los métodos de la «preparación del deportista», además, no dispone de desentrenadores ni las áreas ni medios para llevarlo a la práctica.

9. Los intercambios de experiencia entre los entrenadores de los diferentes deportes en torno al tema estudiado son insuficientes y de pobre sistematicidad en Santiago, por tanto, es muy azaroso superarlos en esa dirección.

Análisis e interpretación (criterios negativos)

Las opiniones divergentes de los metodólogos no se enfocaron a ultranza a oponerse al desentrenamiento y a todos los obstáculos existentes entre la idea de su puesta en práctica definitiva y su materialización, por eso, el desconocimiento expresado por los metodólogos acerca de la proyección nacional sobre el impacto negativo en la población de ex-deportistas, al no haberse incorporado a un programa capaz de garantizar su salud post-deportiva.

La muestra lamentó no haber estudiado la teoría del desentrenamiento en su formación profesional. Incluso, vieron como un freno en el pregrado y posgrado la pobre presencia de ese tema, a pesar de su creciente reconocimiento en el deporte. Eso justifica la insuficiente bibliografía y las pocas investigaciones científicas realizadas al respecto. Esa situación según apuntaron, es una tarea 
pendiente para el Inder y las Facultades de Cultura Física.

Los criterios negativos explicaron en cierta medida la carencia de una concepción coherente, orientada a propiciar una mejor estructuración del sistema de «preparación del deportista» cubano; coincidieron en reconocer el valor de incrementar el trabajo científico, en pos de reducir los años de desconocimiento teórico y práctico en torno al desentrenamiento, en un país donde el deporte es pasión y parte de su cultura, pero por sobre todo, como una cuestión ética y humanista, exige cuidar siempre la salud de sus ex-deportistas, después de entregarse en cuerpo y alma a entrenar bajo un régimen de férrea disciplina y privaciones por muchos años, para representar a la nación con dignidad en las más importantes competencias del mundo.

\section{Resultado 3: criterios interesantes}

1. El desentrenamiento es un tema novedoso y actual, pero necesita mucha atención por los profesionales de la Cultura Física.

2. Los saberes existentes aunque no suficientes, han permitido descubrir su validez para fortalecer el trabajo en la «preparación del deportista».

3. Los conocimientos sobre el desentrenamiento socializado entre los metodólogos, ayudó a ver a la «preparación del deportista» más allá del hecho de entrenar al deportista, para obtener una medalla olímpica o mundial. Es también preocuparse por preservar su salud después del retiro.

4. Ha sido una nueva experiencia, hasta ahora oculta al saber colectivo como profesionales del deporte. Es real. Los ex-deportistas necesitan ser desentrenados si quieren disfrutar de una vejez con mayor calidad de vida.

5. La idea de re-estructurar la visión actual del proceso de «preparación del deportista», supera a la reducida ofrecida durante la formación profesional.

6. El tema es muy interesante, es un proyecto en marcha, lo necesita Santiago de Cuba y el país.

7. Ya no hay dudas: ese proceso al oponerse a los efectos del entrenamiento, proteger la salud $y$ la vida de los ex-deportistas.

8. La idea de tener un centro solo para esa función o varios en la provincia Santiago de Cuba es novedosa, pero esa debe pasar primero por los directivos del deporte en todos sus niveles.

9. Cuandose hablede desentrenamiento, no puede dejarse a un lado, la proyección de la superación de los profesionales de los deportes y de concientizarlos en sus obligaciones de contribuir a proteger la salud de sus deportistas en todas sus etapas.

10. Es interesante aunar todas las fuerzas científicas posibles, para estudiar ese proceso.

11. El éxito llegará cuando se le dé al desentrenamiento tanta importancia como al entrenamiento, pues ambos son subsistemas de la «preparación del deportista». Es interesante, pero hasta ahora solo se ha conocido una cara (entrenamiento) de ese gran proceso, la otra apenas se ha mostrado.

Análisis e interpretación (criterios interesantes)

Los criterios interesantes son estímulos positivos para cualquier proyecto. En este caso los metodólogos al emitir sus juicios sobre el desentrenamiento, reconocieron su novedad y actualidad. Un 39\% del total de respuestas obtenidas vieron con agrado el inicio de la familiarización de ese tema con ellos, pues es un saber no frecuente en el ámbito deportivo de la provincia y la nación. Reconocieron ser esta una nueva experiencia, antes 
ignorada en el debate profesional, a pesar de ocuparse del cómo proteger la salud y la vida de los ex-deportistas.

Hubo comunión de pensamiento, el desentrenamiento primero debía constituirse en una preocupación para todos los directivos del deporte cubano, al ser la principal antítesis del entrenamiento por su valor para los deportistas retirados, por tanto, para los metodólogos resultó muy interesante tratar de aunar todas las fuerzas científicas mediante el trabajo en grupo, para estudiar ese fenómeno en todas sus disciplinas. Todos los criterios interesantes se resumieron en una idea básica: los principales procesos de la «preparación del deportista» son el entrenamiento y el desentrenamiento.

Resultado 4: recomendaciones ofrecidas

1. Indicar en las reuniones de trabajo de todas las comisiones deportivas, empezar a crear conciencia acerca de la necesidad del desentrenamiento. Si no hay cultura sobre eso, de nada sirve ningún esfuerzo al respecto.

2. Crear grupos de especialistas para atender ese tema en Santiago de Cuba. Es una asignatura pendiente para el desarrollo deportivo y profesional en el territorio.

3. Seguir acopiando y socializando todo lo nuevo surgido en torno al asunto tratado.

4. Programarlaseleccióndeentrenadores en cada comisión deportiva, para su capacitación metodológica, teórica y práctica, con el fin de asegurar a sus desentrenadores.

5. Pensar en crear un centro especializado en Santiago de Cuba para el desentrenamiento, donde se reúna el personal adecuado para conducir esa tarea con calidad.

6. Hacen falta proyectos donde se involucren más licenciados y científicos del deporte, con el objetivo de seguir profundizando en su teoría.
7. Mantener la superación profesional al respecto.

8. Crear un ente organizacional con la misión de controlar la aplicación de ese proceso.

9. Avanzar más rápido en la construcción del esquema general del desentrenamiento. Cada año se retiran muchos deportistas y en ese tema muestra pocos avances prácticos.

Análisis e interpretación (recomendaciones)

Las recomendaciones realizadas por los metodólogos, en sentido general, fueron muy importantes, sin embargo, solo se tratarán algunas consideradas representativas. Ellos se pronunciaron por la rápida creación de grupos de especialistas entendidos en el tema del desentrenamiento, capaces de socializar todo el saber creado sobre el mismo. Esta idea es básica, pues el problema no radica en la novedad de ese asunto, sino en cómo atender a las diferentes disciplinas deportivas y sus particularidades cuando se lleve a cabo ese proceso, desde una perspectiva individualizada, en tanto, no existen dos organismos humanos iguales, por ende, esa idea también se expresará en la capacitación los entrenadores por cada comisión, para asumir la dirección de su práctica.

No se necesita formar a un nuevo tipo de profesional de Cultura Física. La proyección es aprovechar las diversas formas de superación como talleres, cursos de posgrado, diplomados y otras, para complementar la preparación recibida por ellos antes de egresar de la educación superior, y aparte de ser entrenadores, lograr ser acreditados como desentrenadores de sus deportes. Otra muy interesante recomendación consistió en mantener la superación de los desentrenadores formados, a través de su participación en proyectos sobre su nueva habilitación, no obstante, este 
aspecto comenzó su materialización, pero su progreso se muestra con lentitud en Santiago de Cuba.

\section{Estructura donde se integran a la «preparación del deportista», el entrenamiento y el desentrenamiento} La fig. 1 es un acercamiento a la interpretación de la teoría de Matveev (1977), relativa a la «periodización del entrenamiento deportivo», ella fue la primera inspiración para la elaboración de una estructura capaz de contener en un mismo espacio como subsistemas, los procesos de entrenamiento y desentrenamiento.

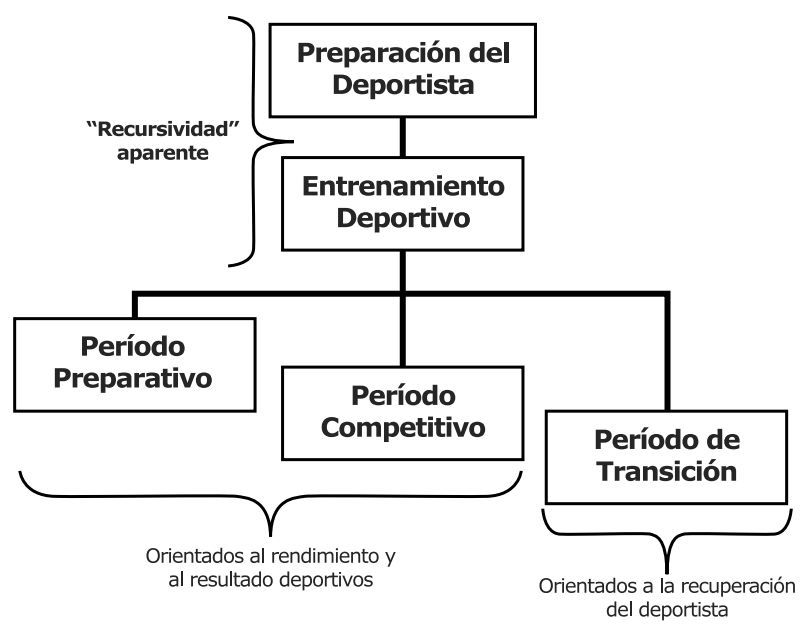

Figura 1. Periodización del entrenamiento deportivo (Matveet, 1977)

El estudio minucioso de la propuesta de Matveev (1977), la revisión de los resultados de autores como Morales (2016), Pino (2014), Mena, (2014), Mustelier (2014), Pérez y Soria (2014), Mayo (2012), Crusellas (2012), Pérez (2012), Barroso (2010), Mujica (2010), Navarro (2010), Andrade (2009), Carvajal, M. (2009) y otros, así como durante el trabajo de familiarización realizado con los 33 metodólogos a cargo de la dirección de las disciplinas deportivas practicadas en Santiago de
Cuba, permitió no continuar entendiendo al desentrenamiento como un proceso ajeno a la «preparación del deportista», al menos en Cuba, pues este como cualquier otro subsistema debe estar concatenado con otro de orden superior, sobre todo, si comparte igual naturaleza. De hecho, esta idea de por sí, supera a la de otros autores, quienes en ningún caso se plantearon la misma, aunque la trataron como si fuera un ente inconexo, en general, de tal preparación.

Tanto en la periodización de Matveev (1977), como en otros trabajos, la esencial similitud encontrada, radicó en concebir o tratar al «periodo de transición» como la estructura básica para recuperar al sujeto en circunstancias de cese temporal o permanente del entrenamiento. Esto no contradice la idea tradicional del entrenamiento ya exhibida en la fig. 1, incluso con los nuevos sistemas, pues en cualquier caso no dejan de incluir los periodos preparatorios y competitivo.

Si todo sistema se subordina a uno superior 0 es síntesis de otros y se acepta como suposición inicial al desentrenamiento ser parte de la «preparación del deportista», se pensó en concebir definitivamente, salvando las confusiones en las definiciones a la que se refirió Matveev (1977), ahora se reconoce en la estructura creada como el "sistema mayor", haciéndolo afín con el entrenamiento y el desentrenamiento como sus subsistemas.

En todos los sistemas, las funciones de sus componentes deben contribuir al logro de sus metas. Eso se muestra contradictorio en la propuesta de Matveev (1977), porque en ese sentido, la contribución del «período de transición», parece activarse, luego de haberse cumplido los objetivos de los períodos preparatorio y competitivo. Esto dificulta la comprensión sistémica de su constructo, sin embargo, 
la idea de favorecer al menos, como tránsito al macrociclo (otra estructura derivada de aquella propuesta) siguiente. Esto aquí se consideró muy positivo, pero aclarando que sus medios no son típicos para elevar la capacidad de rendimiento del deportista. Estas lucubraciones condujeron a los autores de este artículo con una nueva visión al respecto y aprovechando el propósito del «período de transición», replantearse una estructura que englobara a los dos procesos o subsistemas más importantes -según opiniones propiasde la «preparación del deportista». Lo segundo, favorecer su análisis sistémico.

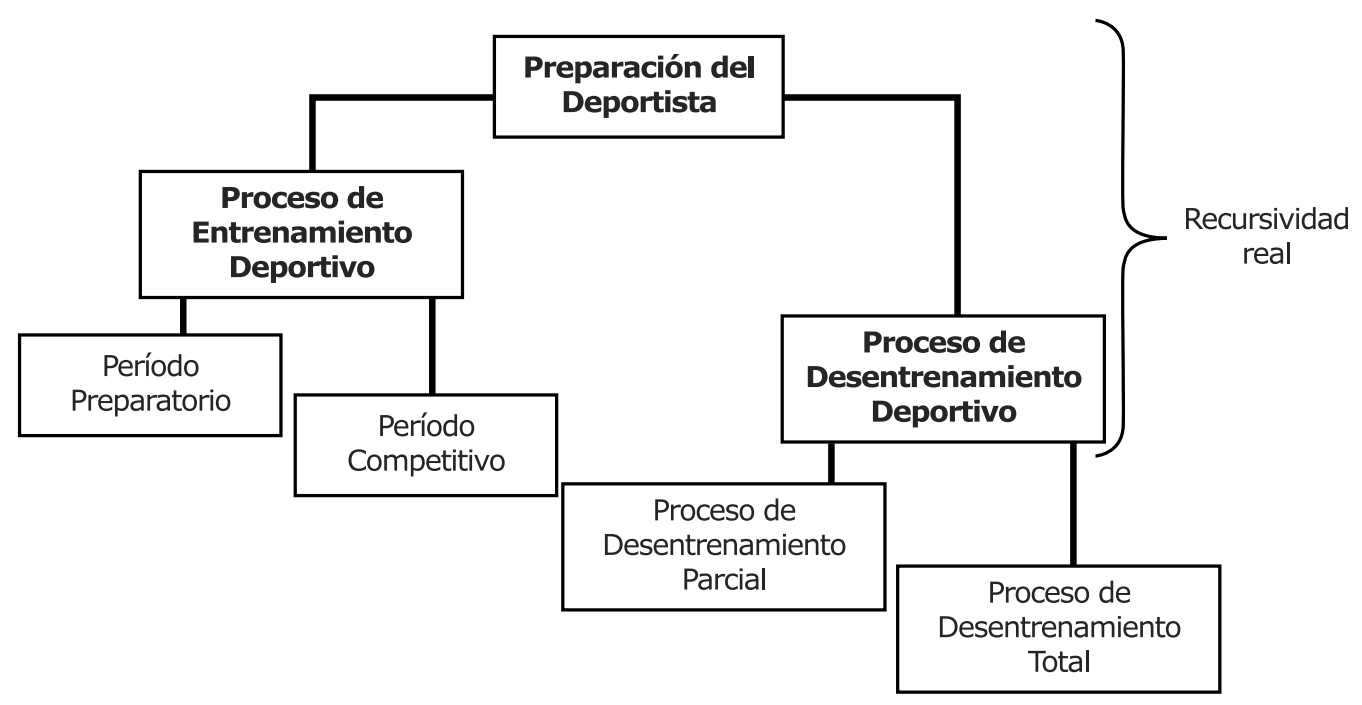

Figura 2. Estructura sistémica donde se integran los procesos de entrenamiento y desentrenamiento.

Tomando en cuenta esas premisas se elaboró la estructura mostrada en la fig. 2. Esta supera a la idea de Matveev (1977), en primer lugar, porque se aprecia, vista la «preparación del deportista» como sistema de orden mayor al menos está conformado por dos procesos, los cuales mantienen relaciones entre sus componentes subordinados y de alguna manera, con los concomitantes. Seaprecia con claridad el nivel de jerarquización y relaciones de dependencia.

Obsérvese como en el subsistema de entrenamiento se desagregó el «período de transición», para reaparecer en la estructura incorporada a la «preparación del deportista» con una nueva identidad: proceso de desentrenamiento parcial.
Esta ubicación es más propia de acuerdo con su función, la cual ahora sí contribuye al cumplimiento de la meta del su subsistema mayor porque todos poseen la misma orientación. El desentrenamiento total lo complementa, pues ambos se caracterizan por concentrar los medios comunes para en general lograr la recuperación transitoria o permanente del deportista, todo depende si continúa o si se retira definitivamente del deporte. En resumen, la estructura de transición de Matveev (1977), no se elimina, sino se reubica en el subsistema concomitante con el entrenamiento, respetándose su idea original básica.

En la fig. 2, quedó redimensionada la «preparación del deportista», porque, por 
un lado, concentra los medios, métodos y procedimientos para elevar la capacidad de rendimiento del deportista, apegada al respeto de sus principios, reglas, orden, armonía y la interdependencia de sus partes, subordinadas a objetivos para lograr un resultado en cierto tiempo previsto. Por otro lado, desde ese mismo gran proceso se puede facilitar la recuperación física y psicológica para re-potenciar el entrenamiento, o tal vez, desestimular las adaptaciones orgánicas sufridas de modo definitivo si se trata de un deportista que pasó al retiro. En otras palabras, desde ese proceso es posible preparar a un deportista para concurrir a una confrontación nacional o internacional, o en un caso puntual, readaptarlo a la vida habitual de la población común con la calidad de vida, y ocuparlo en un centro laboral, sin tener algunas de las enfermedades diagnosticadas en sus estudios por Aguilar (2009), Carvajal (2009), Díaz (2005) y Barrizonte y otros (2003), como isquemia cardiaca, hipertensión arterial, hepatitis, obesidad y taquicardia, en exdeportistas cubanos no desentrenados.

Es incumbencia total del proceso de «preparación del deportista» como sistema de orden mayor, contemplar una estructura destinada de llevar a la "normalidad" al sujeto sometido al trabajo con grandes cargas físicas y psicológicas, para competir al más alto nivel y vencer las exigencias del deporte del siglo XXI. Además, por ejemplo, si tiene en su interior un subsistema con métodos propios para retar tiempos y marcas (entrenamiento), también debe poseer otro para contrarrestar sus efectos (desentrenamiento), porque sería contraproducente 0 erróneo endilgar al IMD la responsabilidad del desentrenamiento mediante la resolución no 82 del Inder (2015), cuando sus métodos no son pedagógicos. Todavía esa idea no ha sido aclarada, aun así, su participación en el proceso mayor es de suma importancia, para cumplir los dos principales objetivos de sus subsistemas.

La medicina del deporte tiene infinidad de métodos para tratar de mantener el equilibrio salud-enfermedad, pero no para entrenar a un deportista hasta alcanzar la cumbre olímpica. Eso le corresponde a la Teoría y Metodología del Entrenamiento Deportivo. Desde esta perspectiva, entonces, no hay dudas, su misión es contribuir a la reincorporación del practicante 0 al entrenamiento o a la vida normal de su comunidad si trata de alguien acogido al retiro, a través de su inserción a algún programa de desentrenamiento, asegurado por los principios y métodos contenidos en la Teoría y Metodología del Desentrenamiento Deportivo, si esta ya se hubiera desarrollado. Por eso, no quedan dudas, quien debe encargarse en principio de esa tarea no es un médico, y sí un desentrenador, poseedor del título de Licenciado en Cultura Física, porque los métodos empleados para el entrenamiento de ese deportista fueron los de su profesión, pero necesita del auxilio de médico del deporte, psicólogos y todos los especialistas demandados por ese programa.

Por último, la «preparación del deportista», se ha tratado de identificar con un sistema, sin embargo, una de las limitaciones, sin profundizar mucho en las ideas de Bertalanffy (1986), era la posibilidad de justificar su recursividad al ser esta aparente. En el modelo tradicional de Matveet (1977) se observaban solo dos elementos con una relación de dependencia: la preparación del deportista y el entrenamiento con todas sus partes. Esto es un error, un sistema estará siempre integrado por al menos, dos subestructuras subordinadas de manera directa. Esto no se cumplía en el caso analizado, y en este artículo se le 
dio solución por vez primera, a pesar de no existir antecedentes de este resultado.

Cuando se dice recursividad aparente en esa concepción sistémica, es porque sí asume a la preparación del deportista como sistema mayor, entonces, solo a este le corresponde un subsistema, por ello, es válido, aunque constituya un error, aceptar la existencia de una igualdad inusitada entre ambas estructuras, y es por ahí donde se escapa la idea de estar en realidad frente a constructo con tal relevancia.

\section{Conclusiones}

1. El desentrenamiento tiene su origen en la periodización del entrenamiento deportivo, pues su célula básica se encuentra en la introducción de momentos de descenso de los valores del volumen y la intensidad, para favorecer la recuperación del deportista.

2. Se explicitó que la práctica del desentrenamiento deportivo en Cuba, se ve afectada primero por no existir una clara visión de este dentro del proceso de preparación del deportista, segundo, pobre conciencia de su necesidad para garantizar una calidad de vida adecuada a quienes se retiran del deporte activo y tercero, por no ser una línea de trabajo de los metodólogos de los deportes de la provincia Santiago de Cuba y por extensión del resto del país.

3. El desentrenamiento deportivo es otro de los procesos, propios de la «preparación del deportista», que requiere de los métodos particulares contenidos en esta y no de otra esfera distinta a esta. Los métodos de la medicina y de la psicología son sus principales complementos.

4. Concebir la inclusión de la estructura o componente del proceso de desentrenamiento dentro de la «preparación de deportista» cubano, permite redimensionar su valor como sistema, al poder compartir el mismo espacio con el proceso de entrenamiento, además deja abierto el espacio a nuevas indagaciones para precisar cuáles serían al menos los medios, métodos, procedimientos cuando se trate del desentrenamiento parcial o total.

\section{Referencias bibliográficas}

Aguilar, E.M. (2009). Metaprograma de entrenamiento físico para el desentrenamiento en atletas elites. [Tesis doctoral no publicada]. Universidad de Ciencias de la Cultura Física "Manuel Fajardo". La Habana.

Alonso, R. (2000). La Medicina Deportiva en el Entrenamiento Deportivo (III). Desentrenamiento Deportivo: Teoría o Hipótesis. Disponible en: http:// www. efdeportes.com/efd40/princ. htm. (Visitado enero 20 de 2016).
Alonso, R.F., Forteza, A. y López, R. (2001). Principios metodológicos del desentrenamiento deportivo. Buenos Aires. EFDeportes.com. [Revista Digital]. Año 7. No 40. Disponible en: http://www.efdeportes.com/. Consultado: 23 mayo 2016

Andrade, J. (2009). Desentrenamiento. Disponible en: http://www. deportesrivera.com.uy/ columnas/ desentrenamiento.php (Consultado en enero 12 de 2016) 
Barrios, J. y Ranzola, A. (1998). Manual para el deporte de iniciación y desarrollo. La Habana: Deportes

Barrizonte, F., y otros. (2003). El desentrenamiento deportivo. [Revista Medicina del Deporte y la Cultura Física]. Instituto de Medicina del Deporte. La Habana

Barroso, M. (2010). Indicadores para la caracterización del retiro deportivo en el atletismo de alto rendimiento: un criterio para la individualización del desentrenamiento. [Tesis doctoral no publicada]. Universidad de Ciencias de la Cultura Física "Manuel Fajardo". Facultad de Cultura Física. Villa Clara

Bertalanffy, L.v. (1986). Teoría General de los Sistemas. Fundamentos, desarrollo y aplicaciones. México, D.F.: Fondo de Cultura Económica

Bompa, T.O. (s.a). Periodización de la fuerza la nueva onda en el entrenamiento de la fuerza. Grupo sobre entrenamiento. Disponible en: www.sobreentrenamiento.com

Carvajal, M. (2009). Desentrenamiento deportivo parcial: una alternativa para entrenadores del área de velocidad. [Tesis de maestría no publicada]. Universidad de Ciencias de la Cultura Física "Manuel Fajardo". Facultad de Cultura Física. Santiago de Cuba

Crusellas, A. (2012). Desentrenamiento deportivo de los atletas retirados. [Tesis de especialidad de posgrado no publicada]. Pinar del Río. Universidad de Ciencias de la Cultura Física "Manuel Fajardo". La Habana

Díaz, A.C. (2005). Folleto de desentrenamiento personalizado, medio ambiente y deporte sostenible. Centro Provincial de Medicina del Deporte. Villa Clara
Ferreiro, R. (2003). Estrategias didácticas del aprendizaje cooperativo. El constructivismo social: una nueva forma de enseñar y aprender. México: Trillas

Harre. D. (1989). Teoríadelentrenamiento deportivo. La Habana: CientíficoTécnica

Inder. Cuba. (2015). Procedimiento de desentrenamiento deportivo para los atletas de alto rendimiento vinculados al Movimiento Deportivo Cubano. Resolución No 82/2015

IMD. Cuba. (2000). Programa Nacional para la Atención a Ex-deportistas. Instituto de Medicina del Deporte La Habana

Matveev, L.P. (1977). Periodización del entrenamiento deportivo. Delegación Nacional de la Educación Física y el Deporte. Madrid. Instituto Nacional de Educación Física

Mayo, M. (2010). Principios para la individualización del desentrenamiento deportivo. [Tesis doctoral no publicada]. Universidad de Ciencias de la Cultura Física "Manuel Fajardo". Facultad de Cultura Física. Villa Clara

Mena, O. (2014). Modelo teóricometodológico para la organicidad del desentrenamiento deportivo. [Tesis de doctorado no publicada]. Universidad de Ciencias de la Cultura Física "Manuel Fajardo". Facultad de Cultura Física. Cienfuegos

Mujica, I. (2010). El desentrenamiento (o qué ocurre cuando dejamos de entrenar). Disponible en: http:// www.inigomujika.com/2010/04/ el-desentrenamiento-o-que-ocurrecuando-dejamos-de-entrenar/344 (Sitio visitado 12 de enero de 2017) 
Mustelier, A. (2014). Acciones educativas encaminada al período de tránsito de los jokistas juveniles. [Trabajo de diploma no publicado]. Universidad de Ciencias de la Cultura Física "Manuel Fajardo". Facultad de Cultura Física. Santiago de Cuba

Morales, C. (2016). Un paso superior para el desentrenamiento deportivo. Medicina Deportiva. [Periódico digital Jit: actualidad del deporte]. Publicación del Inder. La Habana Disponible en: http://www.jit.cu. Consultado: 31 de abril 2016

Navarro, J. (2010). Estado actual del desentrenamiento deportivo de deportistas retirados del fútbol en el municipio Santa Clara. [Trabajo de diploma no publicado]. Universidad de Ciencias de la Cultura Física "Manuel Fajardo". Facultad de Cultura Física. Villa Clara

Ozolin N.G. (1970). Sistema contemporáneo del entrenamiento deportivo. Moscú: FiC
Pérez, T. y Soria, F. (2014). Plan de desentrenamiento deportivo para Levantamiento de Pesas. [Tesina no publicada]. Universidad de Ciencias de la Cultura Física "Manuel Fajardo". Facultad de Cultura Física. Santiago de Cuba

Pérez, Y. (2012). La individualización del entrenamiento y el desentrenamiento deportivo. Multimedia para la superación profesional. [Trabajo de diploma no publicado]. Universidad de Ciencias de la Cultura Física "Manuel Fajardo". Facultad de Cultura Física. Villa Clara

Pino, P. (2014). Informe Anual del Instituto de Medicina del Deporte. Balance Nacional de la Atención a Atletas. La Habana

Platonov, V.N. (1987). Teoría del deporte: manual para los institutos de cultura física. Kiev. Escuela Superior

Verkhoshansky, Y. (2002). Teoría y Metodología del Entrenamiento Deportivo. Colección Entrenamiento Deportivo. Barcelona: Paidotribo 